Historic, Archive Document

Do not assume content reflects current
scientific knowledge, policies, or practices. 



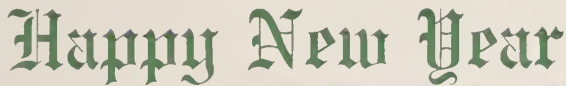

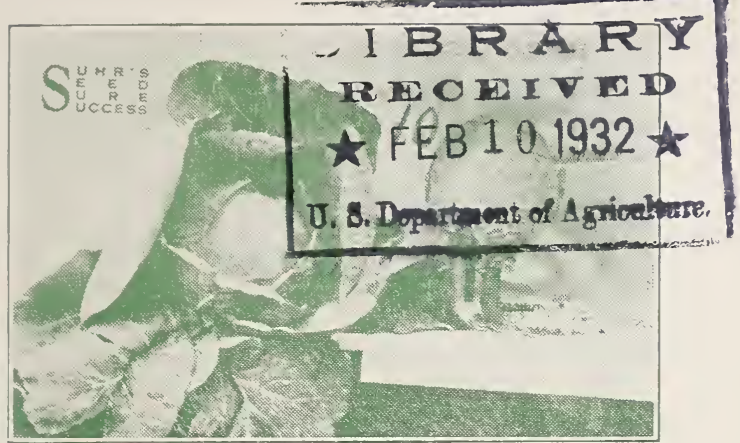

Suhr's Early Copenhagen Market Stock No. 10

\section{SEED OF KNOWN ORIGIN}

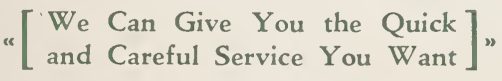

For the benefit of the many seed dealers who have not contracted as heavy this year as usual-

\section{PLEASE KEEP IN MIND}

that we always have first class sto:ks right here in New York; that we can always give you a worthwhile price; that we can always effectuate your orders the very same day we receive them; and that we appreciate your business.

\section{WIRE SUHR FOR YOUR}

$$
\text { SPOT ORDERS }
$$

AND SEE HOW DELIGHTED WE ARE TO SERVE YOU.

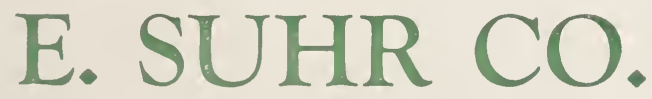

Einar Suhr-John Mosbell

GARDEN SEED GROWERS AND MERCHANTS Wholesale Exclusively

\section{WASHINGTON STREET}

NEW YORK, N. Y.

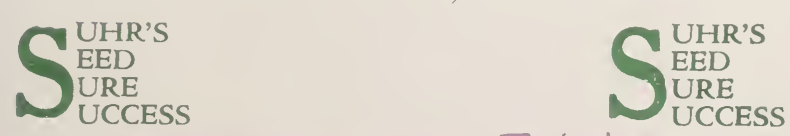




\section{SUHR'S QUALITY GARDEN SEED}

\section{CAULIFLOWER}

Danish Giant or Dry Weather.............. \$10.50

Express Snowball “J. \I." Originator........... 9.50

Earliest Erfurter "T. Suhr".................. 9.00

Superior Snowball "H. Suhr".................. 9.00

Self-protecting Snowball "E. Suhr"........... 8.50

Late Snowball ....................... 8.50

\section{GREEN SPROUTING BROCOLLI}

Calabrese Improved No. $1735 \ldots \ldots \ldots \ldots \ldots \ldots \ldots .4 .50$

\section{CABBAGE (First early varieties)}

Golden Acre .......................... 1.20

Express Ditmarsker (round) ................ 1.00

Early Copenhagen Market.................. .90

Erstling (earliest pointed) .................. .80

Early Jersey $\mid 1$ akefield....................... .75

Charleston VIakefield ....................... .75

(Second early varicties)

Glory of Enkhuizen........................ .80

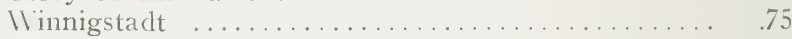

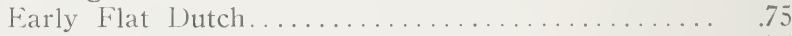

All Head Early...................................

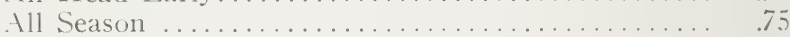

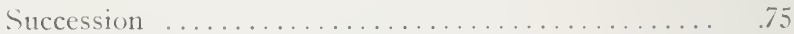

\section{(Late varieties)}

I) anish Ballhead (tall stem) $\ldots \ldots \ldots \ldots \ldots \ldots \ldots$ sold

Danish Ballhead (medium stem) ................ 95

Wanish Ballhead (short stem) ................ . .90

J)anish Roundhead ( (hort stem) . . . . . . . . . . . .95

Late Flat Dutch....................... 90

Certified Penn State Ballhead (original stock)...... 3.00

(Provided with sealed certificate)

\section{CABBAGE SAVOY}

American Drumhead Improved............... 75

lertus ................................. sold

\section{CABBAGE-RED}

Danish Stonehead .......................... 1.50

Mammoth Red Rock........................ 1.50

\section{CABBAGE-CHINESE}

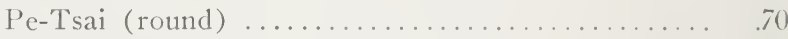

Pe-Tsai (long pointed) ................... .75

\section{BRUSSELS SPROUTS}

Long Island Improved...................... 9.

\section{KALE}

Dwarf Green Curled or Scotch .................. . .35

Blue Curled Siberian..................... $\quad .35$

\section{SPINACH}

King of Denmark....................... $\quad .16$

Bloomsdale Saroy Reselected.................. . .11

Bloomsdale Savoy Long Standing................ . .14

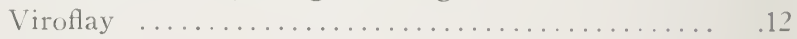

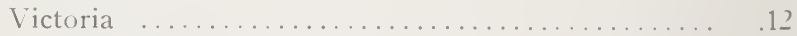

New Zealand $. . . \ldots \ldots \ldots \ldots \ldots \ldots \ldots \ldots . . . \ldots \ldots$

Mustard Spinach (tendergreen) .............. .19) 


\section{GARDEN SEED-Continued}

\section{BEETS (Table)}

Detroit Dark Red.....................\$ .22

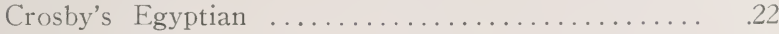

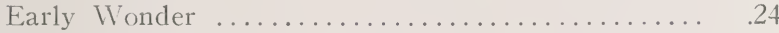

Edmond's Blood Turnip................... 24

\section{SWISS CHARD}

Lucullus

\section{PARSLEY}

Plain $\ldots \ldots \ldots \ldots \ldots \ldots \ldots \ldots \ldots \ldots \ldots \ldots \ldots \ldots . . . \ldots \ldots$

Champion Moss Curled................... 32

TURNIP

Purple Top White Globe................... 18

Purple Top Strap Leaf..................... 18

Early White Flat Dutch.................. 18

Snowball ......................... 18

Purple Top Milan........................ 24

Shogoin (Imported Far East Stock)............ 25

Small Flat White (oriental) ................ 30

\section{RUTA BAGA}

American Purple Top Improved............... .18

I.)anish Bangholm ................... 18

\section{RADISH}

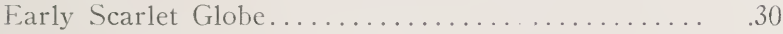

Early Scarlet Turnip (white tip) .............. 28

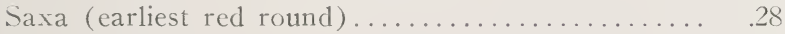

French Breakfast .................... 28

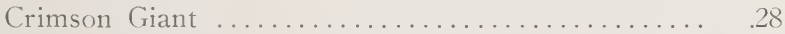

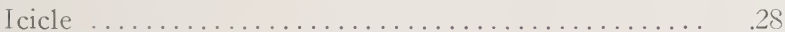

Sparkler (half red, half white) ............... 28

Late 11 inter Mammoth (white) .............. .28

\section{KOHL RABI}

Early IIhite Ticnna..................... 80

\section{LEEK}

American Flag ....................... 75

Musselburgher $\ldots \ldots \ldots \ldots \ldots \ldots \ldots \ldots \ldots \ldots \ldots \ldots \ldots \ldots \ldots \ldots \ldots .75$

CARROT

Chantenay ........................ 42

Danvers Half Long...................... 42

Oxheart $\ldots \ldots \ldots \ldots \ldots \ldots \ldots \ldots \ldots \ldots \ldots \ldots .40$

\section{PARSNIP}

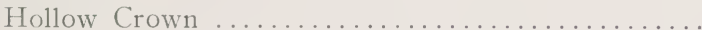

MANGEL WURZEL

Mammoth Long Red.......................... ${ }_{.18}$

Golden Tankard ...................... 18

Danish Sludstrup ....................... 18

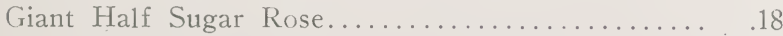

Giant Half Sugar 11 hite................. sold

\section{ONION}

Crystal IIax (Canary Island Grown) . . . . . . . . . . 1.50

Yellow Bermuda (Canary Island Grown) . ......... 1.50

Sweet Spanish Valencia (Spanish Grown)..... 2.50 Superior Bunching ................... 1.25 


\section{SUHR'S QUALITY FLOWER SEED}

ASTER

per ll.

Chrysanthemum Flowered (I)warf) Finest Mixed... \$4.50

Victoria, Finest Mixed..................... 3.50

Ostrich Plume Finest Mixed.................... 2. 2.25

Comet Giant, Finest Mixed..................... 2.25

American Branching, Finest Mixed............... 2.00

CALENDULA

Officinalis F. P1. Balls Orange or Yellow........... .75

HELICHRYSUM (Straw Flower)

Monstr. Fl. Pl., finest double mixed............ 1.60

Monstr. Scarlet, Yellow, Red or Pink (separate colors) 2.75

MYOSOTIS (Forgetmenot)

Alpestris Indigo Dark Blue.

8.00

PANSY

Giant Trimardeau (finest stock), dark purple, white

and rose, blue, yellow, black, white........... 8.90

Giant Trimardeau (finest mixed) ............ 7.25

Giant V arieties, dark shades (our very special stock) 29.50

Giant Roggli (finest mixed) ................. 35.00

Sulhr's large flowering bedding mixture.......... 4.85

\section{SCABIOSA}

(Wonderful range of colors)

Atropurpurea Major Grand Fl. Pl. Mixed.......... 1.50

\section{MARIGOLD}

African (Erecta F1. P1. Tagetes) ................ 1.40

French Dwarf̣ (Patula Nana) Fl. Pl. Mixed....... 1.45

ZINNIA

Elegans Mammoth Fl. Pl. Giants.............. 2.90

NASTURTIUM

Finest Tall Mixture.......................5

Finest Dwarf Mixture....................... .60

\section{LAWN GRASS MIXTURES} BULK ONLY

SUHR'S STANDARD . . . \$20.00

SUHR'S SHADY . . . . . $\$ 40.00$

Two Mixtures suited for your

Critical Customers Only

SAMPLES UPON REQUEST 


\section{LET US QUOTE YOU OUR} LOW 1932 AND 1933 CROP CONTRACT PRICES

WE ARE ALWAYS READY TO SERVE YOU. RUSH ORDERS ARE EFFECTUATED THE VERY

SAME DAY THEY ARE RECEIVED

T H E B E T

R E G A R D L E S S

OF P R I C E

IF YOU WANT TO SELL SEED OF KNOINN ORIGIN

WITH SAFETY AND A WORTHWHILE PROFIT MARGIN

\section{USE}

\section{SUHR'S STOCK}

STOCKS ARE ALIVAYS READY HERE IN NEW' YORK AND REMEMBER THE QUICK, ECONOMICAL AND SAFE IVAY SHIPMENTS WILL REACH YOU

S P E C I A L P R I C E S

WHERE QUANTITIES WARRANT IT

«一

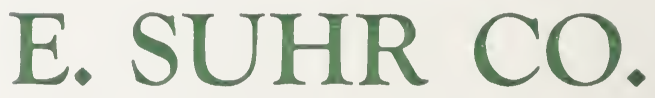

Einar Suhr-John Mosbell

Wholesale Exclusively

23 WASHINGTON STREET

NEW YORK, N. Y. 


\section{WE SERVE THE CRITICAL SEED DEALER}

\section{ALL PRICES ARE:}

F. O. B. NEIV YORK.

DUTY PAID BY US.

PACKING WILL BE CHARGED AT COST.

\section{DELIVERY:}

WHENEVER YOU WANT IT. WILL HOLD SEED FOR YOU UP TO MARCH FIRST, 1932, AND INVOICE FROM DATE OF SHIPPING. TAKE ADVANTAGE OF NOW PREVAILING LOW PRICES AND BOOK BY ALL MEANS.

\section{PAYMENTS:}

CASH WITH ORDER DISCOUNT $5 \%$.

\section{REGULAR TERMS:}

2\% WITHIN 10 DAYS.

NET AND DUE 30 DAYS.

ARRANGEMENTS CAN BE MADE FOR SPECIAL TRADE ACCEPTANCE TERMS.

ALL ORDERS BOOKED SUBJECT TO THE USUAL NON-IVARRANTY CLAUSE AS ADOPTED BY THE AMERICAN SEED TRADE.

QUOTATIONS SUBJECT TO BEING UNSOLD

$$
\ll-\gg
$$

E. Suhr Co. are Specialist Growers of Cauliflower, Cabbage, Spinach, Turnip, Radish, Mangel and Beet Seed.

Seed Farms, Amager Island Home Office-Copenhagen, Denmark 
